\title{
Hiperplasia suprarrenal congénita
}

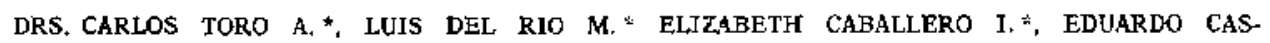
SORLA L." *

\section{COMUNICACION DE DOS CASOS}

InTroducción.- Aun cuando los primeros casos de Hiperplasia Suprarrenal Congénita se describieron en 1887 (1), su estudio se ha profundizado sólo desde 1950, cuando Wilkins y col. demostraron que la cortisona suprimía la excesiva producción de andrógenos y era útil en el tratamiento de estos enfermos (2).

En nuestro medio encontramos publicados 5 casos nacionales $(3,4,5)$, dos de ellos de la forma virilizante simple, dos perdedores de sodio y uno con hipertensión arterial. Esta comunicación se refiere a dos nuevos pacientes, uno de ellos "perdedor de sodio".

Presentación de los casos

Caso No 1, Adrián C. R. Peso de nacimiento 4,250 grs.

Ingresó al mes de vida con signos de deshidratación hipotónica severa, sin otro antecedente que un vómito y una deposición de escaso contenido acuoso en el curso de las 10 hrs. previas.

Un hermano falleció a los dos meses de edad por diarrea aguda, deshidratación y bronconeumonia.

Examen físico.- Lactante de 3.600 grs., signos de deshidratación hipotónica severa. Piel y aréolas mamarias hiperpigmentadas.

Falo de pequeño tamaño; glande descubierto con meato urinario ubicado en posición ventral; bajo él una estructura similar a bolsa escrotal poco desarrollada con fusión total del rafe medio; no se palpó masas en su interior ni en el trayecto de los conductos inguinales. (Fig. 1).

\footnotetext{
* Servicio de Pediatria, Hospital Excquicl González Cortés.
}

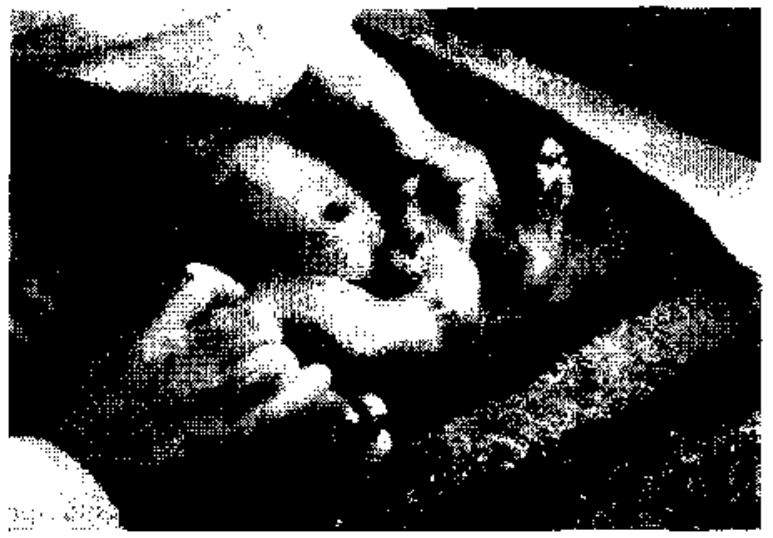

Figura 1: Genitales externos del caso 1.

Presión arterial (método de Flush) 60 y 90 mm. $\mathrm{Hg}$.

\section{Laboratorio}

Sodio plasmático: $135 \mathrm{mEq} \times$ litro; Sodio urinario: 52,1 mEq/L. $/ 24 \mathrm{hrs}$; Potasio plasmático: $7,7 \mathrm{mEg} /$ litro; Potasio urinario: $23 \mathrm{mEq} / \mathrm{L} / 24$ hrs.; 17 Cetoesteroides urinarios: $6,3 \mathrm{mg} . / 24 \mathrm{hrs}$;; Cromatina sexual en frotis bucal: positiva $22 \%$; 17 Hidroxiesteroides urinarios: $0,98 \mathrm{mg} / 24 \mathrm{hrs}$.

Evolución.- Durante los primeros días persistió la deshidratación hipotónica, aủn cuando recibía hasta $20 \mathrm{mEq}$ de Sodio y $300 \mathrm{ml}$. de agua por $\mathrm{Kg} . / 24 \mathrm{hrs}$. Al $10^{\circ}$ día, establecido el diagnóstico de hiperplasia suprarrenal congénita, (Gráfico 1) se inició tratamiento con hidrocortisona $\mathrm{i} / \mathrm{m}$. $10 \mathrm{mg} . / \mathrm{Kg}$. por $24 \mathrm{hrs}$; a pesar de esta terapéutica no se corrigió el trastorno hidrosalino, agregándose entonces DOCA $2,5 \mathrm{mg}$. i. m. c/ 24 
hrs. por 3 días y $1 \mathrm{mg}$. c/ 24 hrs. por 6 días, en solución oleosa, con lo que se logró estabilizar su hidratación (Gráfico 2).

En coincidencia con el uso de DOCA se observó hipertensión arterial de $140 / 90 \mathrm{~mm} \mathrm{Hg}$. lo que determinó su suspensión sin que se alterase nuevamente ef equilibrio hidrosalino, con excreción urinaria de sodio de $119 \mathrm{mEq} / \mathrm{Lt} / 24 \mathrm{hrs}$. y de potasio de $33 \mathrm{mEq} /$ Lt. $/ 24$ hrs.

El enfermo siguió en buenas condiciones bajo tratamiento con Hidrocortisona i. m. y 2 a 3 gr. diarios de Citrato de Sodio oral hasta el día 50 de hospitalización en que presentó una bronconeumonia falleciendo dos días después.

La autopsia denostró bronconeumonia abscedada bilatera1, supratrenales de 13 gramos con hiperplasia demostrada histológicamente; ovarios, útero y vagina normalmente desarrollados, sin orjficio vaginal externo. (Fig. 2).

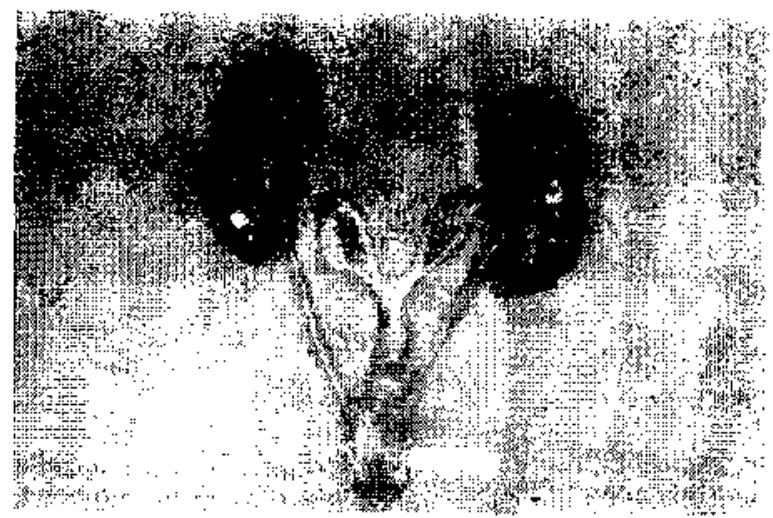

Figura 2: centales microos teincunos y marcado aumento de tanaĩo de ambas suprartenales en el caso $]$

Caso No 2: Verónica C. C. Peso de nacimiento 3.300 grs.

Ingresó a los 8 meses de edad para estudio de genitales ambiguos. 5 hermanos fallecieron en el período de lactante por causas que la madre desconoce.

Examen físico.- Peso 6.500 grs. Talla $66 \mathrm{~cm}$. Labios mayores bien conformados. Labios menores fusionados, terminaban hacia adelante en clítoris de $1 \mathrm{~cm}$. de largo y $0,5 \mathrm{~cm}$. de djámetro en cuya base había un pequeño orificio triangular, y hacia atrás en un segundo orificio circular de $3 \mathrm{~mm}$. que semejaba el introito vaginal. (Fig. 3).

Presión arterial $95 \mathrm{mrm}$. $\mathrm{Hg}$, sistólica.

\section{Laboratorio}

Cromatina sexual $14 \%$ : positiva. Cariograma: Cariotipo $46 \mathrm{xx}$, corresponde a sexo femenino normal. 17 Cetoesteroides urinarios $4,1 \mathrm{mg}$. en 24 hrs. Sodio plasmático $143 \mathrm{mEq} /$ litro. Sodio urinario $40 \mathrm{mEq} / \mathrm{lt}$. $/ 24 \mathrm{hrs}$. Potasio plasmático 5-0 $\mathrm{MEq} / \mathrm{lt}$. Potasio urinario $71 \mathrm{mEq} / \mathrm{lt}$. $/ 24 \mathrm{hrs}$.

Edad ósea radiológica concordante (Greulich y Pyle).

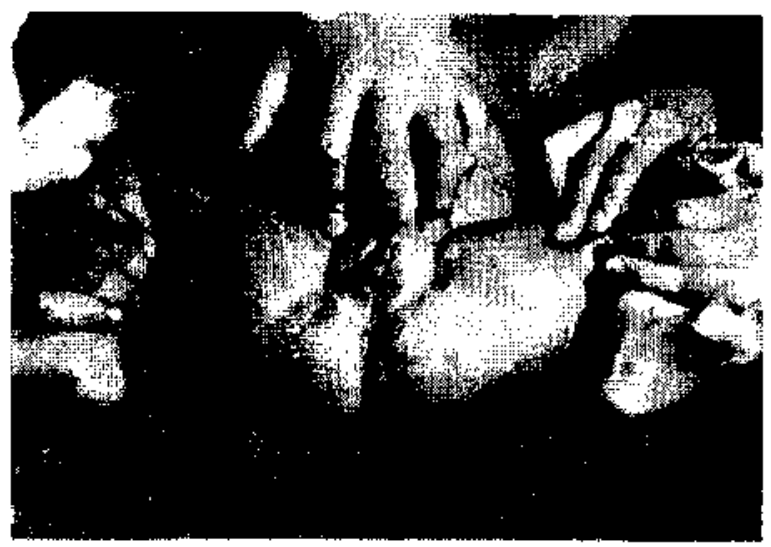

Figura 3.- Genitales extcroos en el caso № 2.

Uretrocistografía: vejiga normal; al retirar el catéter uretral se obtuvo contraste vaginal. (Figura 4).

\section{Evolución.}

Con el diagnóstico probable de Hiperplasia Suprarenal congénita se inició tratamiento con hi-

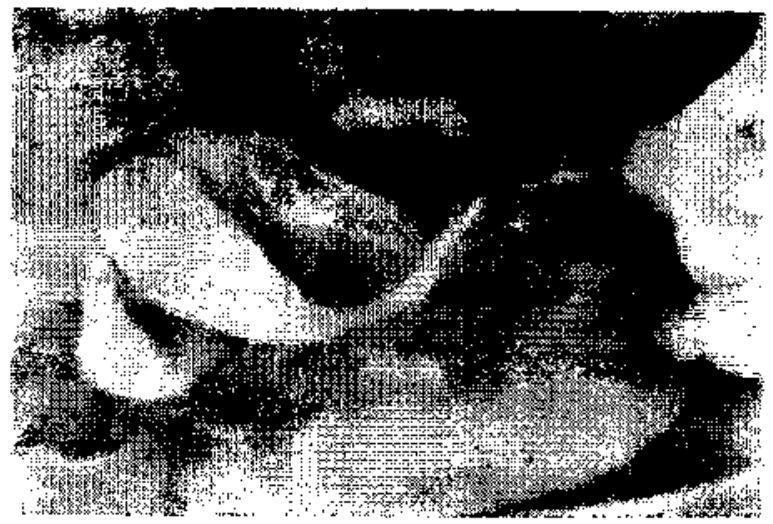

Figura 4: Uretrocistugratia en el casn 2. ninuestra contraste simultáneo de vejiga y vagina.

drocortisona i. m. y 24 hrs. después los 17 cetoesteroides urinarios habían disminuido a $1,1 \mathrm{mg}$. en 24 hrs. Una semana más tarde el examen de control mostró 17 cetoesteroides urinarios de $0,33 \mathrm{mg}$. en 24 hrs. (Gráf. 1 ).

Actualmente se mantiene en buenas condiciones con Hidrocortisona oral y no requiere suplemento dietético de sodio. 
HI PERPL ASIA SUPRARRENAI CONGENITA VARIACION OE LOS 17 CETOESTEROIDES URINARIOS

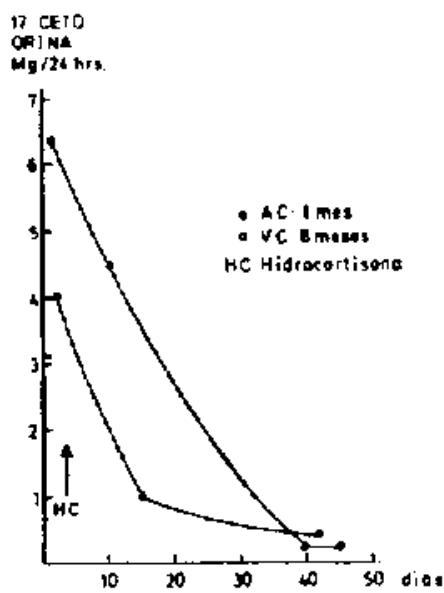

Gráfico 1: Disminución de los 17 Cetcesteruides urinarios cun la administración de hidrocortisona.

Discuslón. Gráfico III. La existencia, en ambos casos, de genitales ambiguos, sexo cromosómico femenino y 17 cetoesteroides urinarios aumentados que se normalizaron con la administración de cortisona fundamentan el diagnóstico de Hiperplasia Suprarrenal congénita.

La deficiencia de 20-22 desmolasa puede descartarse por tratarse de individuos de sexo femenino ccomosómico y anatómico, con marcada virilización y aumento de los 17 cetoesteroides urinarios.

A lo anterior se agrega en el caso jos 17 hidroxiesteroides urinarios normales y la ausencia en la autopsia de las alteraciones lipoides de la

Hospotel E. Gomanle Cortés

Servicio of Rediatrio

Samiogo de Chile-19)2

Grálice 11

EYOLUCION DE LOS ELECTROLITOS PLASMATICOS COH LA ADMINISTRACION QE HIRROCORIISOMA Y DOCA

CAso:

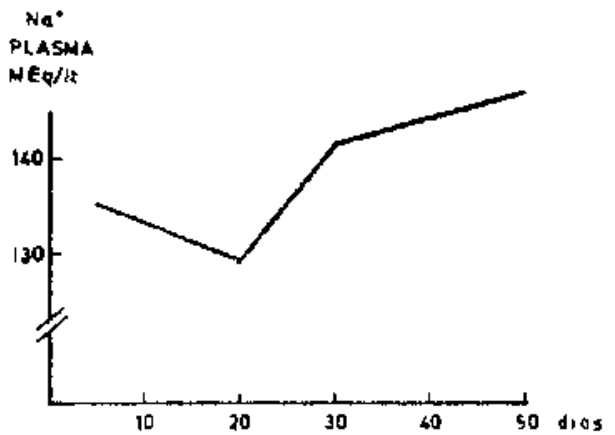

Gráfico 2: Muestra el aumento progresivo del sodio plasmático despues de la administración de DOCA en el dia 20 de evolución. histología suprarrenal, y en el $2^{\circ}$ Jos 17 hidroxiesteroides urinarios aumentados, la ausencia de sindrome perdedor de sodio y la sobrevida.

La marcada virilizacion, y la cromatina sexual positiva hacen improbable el diagnóstico de deficiencia de $3 \mathrm{~B}$ hidroxidehidrogenasa-isomerasa en el caso 1 .

El caso 2 podría tratarse de una forma incompletit del defecto ya que se lo ha descrito sin sindrome perdedor de sodio, sin embargo, a pesar de que no se dispuso de fraccionamiento de los $17 \mathrm{ce}$ toesteroides para detectar eliminación anormal de dehidroepiandrosterona, este defecto no puede postularse por la elevadal excreción de 17 hidroxiesteroides urinarios.

La presencia de sindrome perdedor de sodio en el primer paciente y el aumento de los $17 \mathrm{hi}-$ droxiesteroides urinarios en el segundo, se oponen a un posible bloqueo de la 17 hidroxilasa.

La deficiencia de 21 hidroxilaxa parece lo más probable en estos pacientes, por tratarse del de-

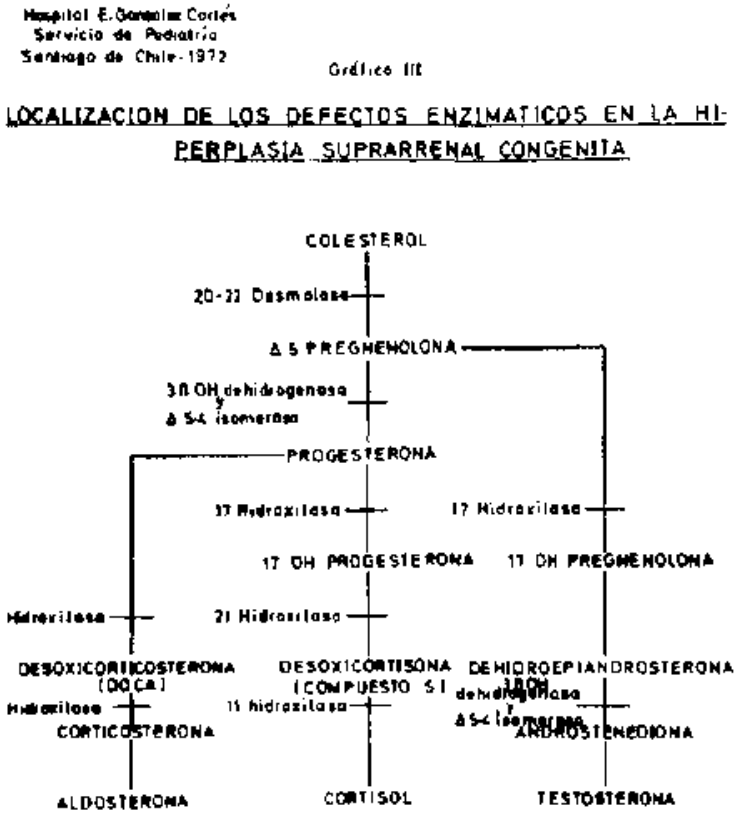

Gráfico 3: Whitación del defecto enzimálico en hiperpiasia suprorresal conginita.

fecto enzimática más frecuente, por las características anatómicas y cromosómicas y la excreción elevada de 17 cetoesteroides urinarios.

En el caso Nọ 1 existía además 17 hidroxiesteroides urinarios normales y un sindrome perdedor de sodio que sólo respondió a la administración de DOCA y requirió aportes adicionales importantes de sodio para lograr el equilibrio hidro-electrolítico. En el $2^{\circ}$ caso no había sindrome perdedor de sodio y los 17 hidroxiesteroides urinarios estaban aumentados, lo que es posible en este tipo de defecto del metabolismo suprarrenal. 
El déficit de 11 hidroxilasas se manifiesta en la mayoría de los casos por hiperaldosteronismo, por lo que puede descartarse en el primer caso en que existía el defecto opuesto. Sin embargo se ban descrito formas sin hjpertensión, con electrolitos plasmáticos normales y 17 hidroxiestcroides urinirios elevados según el método de Porter Silber, luego esta posibilidad no puede eliminarse en el $2^{\circ}$ caso mientras no se determine la excreción urinaria de tetrahidrodesoxicortisol.

\section{RESUMEN}

Se presentan dos casos de Hiperplasia Saprarrenal congénita; se interpreta el primero como un deficit de 21 hidroxilasa en su forma perdedora de sodio, y el segundo como una forma simple del mismo defecto por su mayor frecuencia, o un déficit de 11 hidroxilasa sin hipertensión arterial.

\section{SUMMARY}

Two cases of congenital adrenal hyperplasia are presented, one of then a salt loosing form.

Differential diagnosis is briefly discussed.

\section{BIBLIOGKAE\{A}

I.- Phillips. Citado por Raitis y Newns G. H.: Am. J. Dis. Child. 39: 324, 1964.

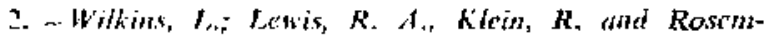
berg, E.: The supression of androgen secretion by cortisone in a case of congenital Advenal hyperplasia. Bull Johe Hopkins Hospital 86: 249, 1950.

3.-Beas, F., Monckeberg, F.: Hermafroditismo e hiperplasia suprarrenal. Pediatría 1: 304, 1958.

4.-Maccioni, A. y Espinoza, J.: Discusión clínica y anátomopatológica de enfermos que presentaron un problema diagnóstico. Pediatría 4: 312, 1961.

3.-Bcas, F,; Jadresic', A.; Abodoswsky, N. y Figueroa, $M .:$ Hiperplasia Suprarrenal virilizante. Rev. Chilena Pediatría 35: 721, 1964.

6.- Gardner Ly H. I. Endoctine and Genetic dis of child hood. V. B. Sauders Co. Philadelphia and London, 1969.

7.- Migeon C. I. en Cooke R. E. The Biol. Busis of Pediatric Practice Mc Graw Hill, New York 1968. P. 1153 Chapt. 118 P. 1153. 